\title{
In memoriam Stephan Perren (1932-2019)
}

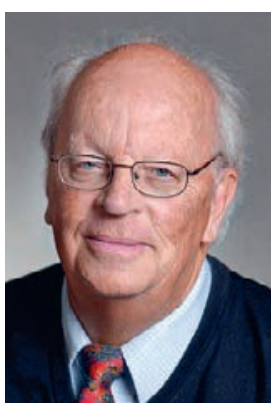

Stephan Perren
Der Lebenskreis von Stephan Perren, einem grossartigen Arzt, Forscher und Freund, hat sich geschlossen. Er starb unerwartet am 21. November 2019.

Stephan ist am 7. Oktober 1932 in Zermatt geboren und aufgewachsen. Nach dem Staatsexamen 1960 an der Universität Zürich folgten Jahre der medizinischen Weiterbildung am Regionalspital Visp (1960/61), an der Frauenklinik Winterthur (1962) und in der Chirurgie des Kantonsspitals Chur, wo er von 1962 bis 1963 sowie 1966 und 1967 unter dem Mitbegründer der AO-Stiftung, Martin Allgöwer, tätig war.

\section{Begeisterter Forscher}

Dazwischen lagen Jahre an der ThurgauischSchaffhausischen Heilstätte Davos (1963/64) und am Labor für experimentelle Chirurgie Davos, heute bekannt als ARI, wo er unter Direktor Herbert Fleisch (1964/65) wirkte. Sein Hang zur Forschung begleitete ihn das ganze Leben. Weltweit bekannt wurde Stephan für seine Dehnungstheorie, welche die Gewebedeformation als kritischen mechanischen Faktor für die Knochenheilung postuliert.

Im Jahr 1967 wurde Stephan als Direktor des «AO Research Institute Davos» (ARI) berufen, das er bis 1996 leitete. In dieser Zeit wuchs das ARI in seiner internationalen Reputation. Stephan wurde gleichermassen

\section{Sein Hang zur Forschung begleitete ihn das ganze Leben.}

Dr. med. Thomas Kehl, MBA

Facharzt

Orthopädische Chirurgie und Traumatologie

Klinikstrasse 4

CH-7272 Davos Clavadel

thomaskehl[at]bluewin.ch als Ikone und Visionär geschätzt wie auch für sein Engagement bei der Ausbildung und Betreuung junger Forscher.

Stephan war Honorarprofessor an der Universität Bern, der Universidad de Montevideo in Uruguay und der University of Wales, Aberystwyth. Im Laufe seiner langen Karriere lehrte er ausserdem als Dozent an der Universität Basel, der ETH Zürich, der Universität Bern und der Hong Kong University und war ein geschätzter Experte für die AO-Kurse in Davos und im Ausland.

\section{Zahlreiche Ehrungen}

Unter den vielen bedeutenden Auszeichnungen, die Stephan im Laufe seines Lebens erhielt, waren unter anderem: der AO-Preis (1968), der Danis-Preis der «Société Internationale de Chirurgie» (1983), die Johann-Friedrich-Dieffenbach-Büste der Deutschen Gesellschaft für Unfallchirurgie (1993), der «Association

\section{Stephan wurde gleichermassen als Ikone und Visionär geschätzt.}

Internationale pour l'Ostéosynthèse Dynamique Award» zum 100. Geburtstag von Gerhard Küntscher (2000), der MD-Preis der Orthopädischen Forschungsgesellschaft Arthur Steindler (2001) und 2004 der AOPreis für sein Lebenswerk.

Als aktives Mitglied des Rotary Clubs Davos Klosters hat Stephan die Idee und das Gedankengut von Rotary beispielhaft im Alltag gelebt und gefördert.

Wie kaum ein Zweiter schaffte es Stephan, Familie, Beruf und Freizeit unter einem Dach zu vereinen. Seine Menschlichkeit, fachlichen Qualitäten und Schaffenskraft machten ihn zu einem grossartigen Menschen und Arzt.

Alle, die ihn kannten, werden Stephan Perren vermissen und ihm ein ehrendes Andenken bewahren.

Thomas Keh 